\title{
Polymorphisms in the mitochondrial oxidative phosphorylation chain genes as prognostic markers for colorectal cancer
}

\author{
Jesus Lascorz ${ }^{1}$, Melanie Bevier ${ }^{1}$, Witigo V Schönfels ${ }^{2}$, Holger Kalthoff ${ }^{3}$, Heiko Aselmann², Jan Beckmann², \\ Jan Egberts ${ }^{2}$, Stephan Buch ${ }^{4,5}$, Thomas Becker ${ }^{2}$, Stefan Schreiber ${ }^{5}$, Jochen Hampe ${ }^{5}$, Kari Hemminki ${ }^{1,6}$, \\ Asta Försti ${ }^{1,6^{*}+}$ and Clemens Schafmayer ${ }^{2,4+}$
}

\begin{abstract}
Background: Currently, the TNM classification of malignant tumours based on clinicopathological staging remains the standard for colorectal cancer (CRC) prognostication. Recently, we identified the mitochondrial oxidative phosphorylation chain as a consistently overrepresented category in the published gene expression profiling (GEP) studies on CRC prognosis.

Methods: We evaluated associations of putative regulatory single nucleotide polymorphisms (SNPs) in genes from the oxidative phosphorylation chain with survival and disease prognosis in 613 CRC patients from Northern Germany (PopGen cohort).

Results: Two SNPs in the 3' untranslated region of UQCRB (complex III), rs7836698 and rs10504961, were associated with overall survival ( $\mathrm{HR}=0.52,95 \% \mathrm{Cl} 0.32-0.85$ and $\mathrm{HR}=0.64,95 \% \mathrm{Cl} 0.42-0.99$, for $\Pi$ carriers). These associations were restricted to the group of patients with cancer located in the colon ( $\mathrm{HR}=0.42,95 \% \mathrm{Cl} 0.22-0.82$ and $\mathrm{HR}=0.46$, $95 \% \mathrm{Cl}$ 0.25-0.83). Multivariate analysis indicated that both markers might act as independent prognostic markers. Additionally, the $\Pi$ carriers were $\sim 2$ times more likely to develop tumours in the colon than in the rectum. Two SNPs in COX6B1 (complex IV) were associated with lymph node metastasis in a dominant model (rs6510502, $\mathrm{OR}=1.75,95 \% \mathrm{Cl} 1.20-2.57$; rs10420252, $\mathrm{OR}=1.68,95 \% \mathrm{Cl} 1.11-2.53)$; rs6510502 was associated also with distant metastasis $(\mathrm{OR}=1.67,95 \% \mathrm{Cl} 1.09-2.56$ in a dominant model).
\end{abstract}

Conclusions: This is the first report suggesting that markers in genes from the mitochondrial oxidative chain might be prognostic factors for CRC. Additional studies replicating the presented findings are needed.

\section{Background}

Colorectal cancer $(\mathrm{CRC})$ is the third most common cancer and the fourth-leading cause of cancer death worldwide [1]. Currently, the TNM classification of malignant tumours based on clinicopathological staging remains the standard for CRC prognostication [2]. Many gene expression profiling (GEP) studies on prognosis of CRC have been performed in the last decade using microarray

\footnotetext{
* Correspondence: a.foersti@dkfz.de

${ }^{\dagger}$ Equal contributors

'Division of Molecular Genetic Epidemiology, German Cancer Research Center (DKFZ), Im Neuenheimer Feld 580, Heidelberg, 69120, Germany ${ }^{6}$ Center for Primary Health Care Research, Clinical Research Center, Lund University, Malmö, Sweden

Full list of author information is available at the end of the article
}

technology, with the aim to identify a gene expression profile to discriminate more aggressive from less aggressive CRC. However, due to their low overlap in the identified genes, no reliable signature useful in the clinical practice has been found. Recently, we published a systematic pathway-based enrichment analysis of 23 independent GEP studies on prognosis of CRC [3]. This analysis indicated the mitochondrial oxidative phosphorylation (OXPHOS) chain as a significantly and consistently overrepresented prognostic category for CRC.

Already several decades ago, it was suggested that impaired oxidative metabolism may cause malignant growth [4]. In cancer cells, there is an enhanced glucose use, slowing the rate of the tricarboxylic acid cycle and

\section{Ciomed Central}


oxidative phosphorylation and increasing glycolysis, as the way to generate energy in form of adenosine triphosphate (ATP), despite aerobic conditions [5]. This assumption, known as Warburg's hypothesis, has been rediscovered by a broad range of experimental approaches showing interaction of mitochondrial metabolism and tumour growth [6-8]. Additionally, germline mutations in the mitochondrial succinate dehydrogenase (complex II of the OXPHOS chain) subunits SDHD, SDHC, and SDHB are a frequent cause of paragangliomas of the head and neck and of phaeochromocytomas [9].

Thus, the OXPHOS chain appears as a promising candidate pathway for CRC progression. The OXPHOS system, whose proteins are encoded by both nuclear and mitochondrial DNA (mtDNA), consists of five major protein complexes named complex I, II, III, IV, and V, localized on the inner mitochondrial membrane. Its main function is the generation of ATP [10]. We therefore selected putatively functional single nucleotide polymorphisms (SNPs) in the genes from the pathway, and investigated their possible role in the progression of CRC and the prognosis of patients in a group of 613 German CRC patients.

\section{Methods}

\section{Study population}

The 613 patients included in the study belonged to the population-based PopGen project in Schleswig-Holstein (Germany) [11]. All individuals were of German origin, i. e. with both parents born in Germany. Patients fulfilling either the clinical Amsterdam or Bethesda criteria for hereditary non-polyposis colorectal cancer (HNPCC) were excluded from the study, as were patients with a history of malignant disease or inflammatory bowel disease. For a full description of the characteristics of the 613 patients see Table 1 and Castro et al. [12]. The work has been approved by the corresponding ethical committees and subjects gave informed consent to the work.

\section{Gene and SNP selection}

Candidate genes in the OXPHOS pathway (map00190 in the KEGG database [13]) were selected based on a recently published meta-analysis of GEP studies on CRC prognosis [3]. Three out of the seven genes reported in two GEP studies had SNPs in the putative regulatory regions (ATP5C1 from complex V, COX5B and COX6B1 from complex IV). Two additional genes with SNPs in putative regulatory regions, each reported in one GEP study, were also included, namely NDUFS2 from complex I and UQCRB from complex III. With these selection criteria, the four complexes from the OXPHOS chain which allow flowing of protons between the mitochondrial matrix and the intermembrane space, resulting into energy generation
Table 1 Characteristics of the 613 CRC patients at the time of diagnosis

\begin{tabular}{|c|c|c|}
\hline Parameter & $\begin{array}{l}\text { Number of } \\
\text { patients }\end{array}$ & $\begin{array}{c}\% \text { of } \\
\text { patients }\end{array}$ \\
\hline \multicolumn{3}{|l|}{ Gender } \\
\hline Male & 325 & 53.0 \\
\hline Female & 284 & 46.3 \\
\hline Unknown & 4 & 0.7 \\
\hline \multicolumn{3}{|c|}{ Age at diagnosis (year) } \\
\hline$<65$ & 341 & 55.6 \\
\hline$\geq 65$ & 272 & 44.4 \\
\hline \multicolumn{3}{|c|}{ Histopathologic grade (G) } \\
\hline G1 & 20 & 3.3 \\
\hline G2 & 478 & 77.9 \\
\hline G3 & 98 & 16.0 \\
\hline G4 & 1 & 0.2 \\
\hline Gx & 16 & 2.6 \\
\hline \multicolumn{3}{|c|}{ Pretherapeutic UICC TNM stage } \\
\hline । & 161 & 26.3 \\
\hline$\|$ & 156 & 25.4 \\
\hline III & 149 & 24.3 \\
\hline IV & 133 & 21.7 \\
\hline Unknown & 14 & 2.3 \\
\hline \multicolumn{3}{|c|}{ Primary tumour size $(\mathrm{T})$} \\
\hline TO & 6 & 1.0 \\
\hline $\mathrm{T} 1$ & 76 & 12.3 \\
\hline $\mathrm{T} 2$ & 138 & 22.5 \\
\hline T3 & 327 & 53.3 \\
\hline $\mathrm{T} 4$ & 58 & 9.4 \\
\hline Tis or Tx & 9 & 1.5 \\
\hline \multicolumn{3}{|c|}{$\begin{array}{l}\text { Regional lymph node involvement } \\
\text { (N) }\end{array}$} \\
\hline N- & 354 & 57.7 \\
\hline $\mathrm{N}+$ & 255 & 41.6 \\
\hline $\mathrm{Nx}$ & 4 & 0.7 \\
\hline \multicolumn{3}{|c|}{ Distant metastasis (M) } \\
\hline M- & 478 & 78.0 \\
\hline $\mathrm{M}+$ & 135 & 22.0 \\
\hline \multicolumn{3}{|l|}{ Localisation } \\
\hline Rectum & 327 & 53.3 \\
\hline Colon & 286 & 46.7 \\
\hline \multicolumn{3}{|c|}{ Adjuvant therapy } \\
\hline Any $^{a}$ & 250 & 40.8 \\
\hline None & 282 & 46.0 \\
\hline Unknown & 81 & 13.2 \\
\hline \multicolumn{3}{|l|}{ Pretreatment } \\
\hline Any ${ }^{b}$ & 68 & 11.1 \\
\hline
\end{tabular}


Table 1 Characteristics of the 613 CRC patients at the time of diagnosis (Continued)

$\begin{array}{ccc}\text { None } & 517 & 84.3 \\ \text { Unknown } & 28 & 4.6\end{array}$

a Comprises radiotherapy $(n=13)$, chemotherapy $(n=86)$, radio-chemotherapy $(n=59)$, immunotherapy $(n=3)$, chemotherapy plus 5FU/FA $(n=68)$, radiochemotherapy plus 5FU/FA $(n=18)$, and other $(n=3)$.

b Comprises radiotherapy $(n=5)$ and radio-chemotherapy $(n=63)$.

in form of ATP, were included in the study. Finally, SNPs in the genes GAPDH and HSPD1 were also selected, since both genes might define the cellular bioenergetic activity of the mitochondria [6].

SNPs within the set of candidate genes (Table 2 and Additional file 1: Table S1) were selected according to the following criteria: minor allele frequency (MAF) $>0.05$ in HapMap CEPH population (Utah residents with ancestry from northern and western Europe - CEU), and location in potentially regulatory gene regions $\left(5^{\prime}\right.$ and $3^{\prime}$-UTR, promoter or non-synonymous coding SNPs). In regions with several SNPs in high linkage disequilibrium (LD), tagSNPs were selected for genotyping. LD between the SNPs was calculated using Haploview [14]. The promoter SNP rs2802460 in ATP5C1 was reported to regulate expression of the gene in lymphoblastoid cell lines (eSNPs) on a genome-wide association study of global gene expression [15]. Predictions of the possible impact of amino acid substitutions on the structure and function of the protein were performed with PolyPhen2 [16].

\section{Genotyping}

The 613 DNAs used in the study were extracted from peripheral venous blood using the FlexiGene chemistry (Qiagen, Hilden, Germany) according to the manufacturer's protocols. From them, $272(44.4 \%)$ samples were subjected to genome-wide amplification using the illustra GenomiPhi V2 DNA Amplification Kit (GE Healthcare, Freiburg, Germany) according to manufacturer's instructions. Genotyping was performed using $5 \mathrm{ng}$ of either genomic or whole-genome amplified DNA in 384-well plate format, using KASPar assays on demand (KBiosciences, Hoddesdon, UK) and following the manufacturer's protocols. Average genotyping call rate was $98.8 \%$. All polymorphisms were in Hardy-Weinberg equilibrium.

\section{Statistical analysis}

SAS software version 9.2 (SAS Institute) was used in all analysis. Unadjusted association between the genotyped markers and patient characteristics were evaluated by chisquare test. Odds ratios (ORs) with 95\% confidence intervals (CIs) were estimated using logistic regression. Effect of the different genotypes on CRC survival was evaluated using Kaplan-Meier method and was compared using logrank testing. Analysis of different parameters for prognostic significance was done by univariate and multivariate Cox proportional hazard models. P values $<0.05$ were considered statistically significant. Follow-up time was calculated from the date of CRC diagnosis to the CRC specific death

Table 2 SNPs evaluated in the study

\begin{tabular}{|c|c|c|c|c|c|}
\hline Gene & Complex & SNP & Major/minor allele & Location & MAF \\
\hline NDUFS2 & 1 & rs33941127 & $C / T$ & Promoter & $T-0.21$ \\
\hline NDUFS2 & । & rs3813623 & $\mathrm{G} / \mathrm{T}$ & $5^{\prime}-$ UTR & $T-0.15$ \\
\hline NDUFS2 & । & rs11538340 & $C / A$ & Coding P20T & A - 0.06 \\
\hline NDUFS2 & । & rs11576415 & $C / G$ & Coding P352A & $G-0.09$ \\
\hline NDUFS2 & । & rs1136224 & $A / G$ & $3^{\prime}-$ UTR & $G-0.17$ \\
\hline UQCRB & III & rs7836698 & $C / T$ & 3'-UTR & $T-0.42$ \\
\hline UQCRB & III & rs10504961 & $\mathrm{C} / \mathrm{T}$ & $3^{\prime}-$ UTR & $T-0.50$ \\
\hline COX5B & IV & rs11904110 & $\mathrm{T} / \mathrm{C}$ & Promoter & $C-0.06$ \\
\hline Cox6B1 & IV & rs6510502 & $\mathrm{A} / \mathrm{C}$ & Promoter & $C-0.13$ \\
\hline COX6B1 & IV & rs10420252 & $\mathrm{G} / \mathrm{A}$ & $5^{\prime}-U T R$ & A - 0.10 \\
\hline ATP5C1 & V & rs11255367 & $\mathrm{G} / \mathrm{A}$ & Promoter & A - 0.12 \\
\hline ATP5C1 & V & rs 2802460 & $\mathrm{~T} / \mathrm{C}$ & Promoter & $C-0.26$ \\
\hline ATP5C1 & V & rs4655 & $\mathrm{T} / \mathrm{C}$ & 3'-UTR & $C-0.36$ \\
\hline GAPDH & & rs7971637 & $C / T$ & Promoter & $\mathrm{T}-0.19$ \\
\hline GAPDH & & rs1136666 & $C / G$ & 5'-UTR & $G-0.26$ \\
\hline HSPD1 & & rs1116734 & $C / G$ & Promoter & $G-0.33$ \\
\hline
\end{tabular}

Two additional SNPs were genotyped, but showed only one allele in the 613 samples: rs2071038 (COX5B) and rs955 (HSPD1).

$\mathrm{MAF}$, minor allele frequency in the $613 \mathrm{CRC}$ patients investigated; NDUFS2, NADH dehydrogenase (ubiquinone) Fe-S protein 2, 49kDa (NADH-coenzyme Q

reductase); UQCRB, ubiquinol-cytochrome $\mathrm{c}$ reductase binding protein; COX5B, cytochrome c oxidase subunit Vb; COX6B1, cytochrome $\mathrm{c}$ oxidase subunit VIb, polypeptide 1; ATP5C1, ATP synthase, $\mathrm{H}$ + transporting, mitochondrial F1 complex, gamma polypeptide 1; GAPDH, glyceraldehyde-3-phosphate dehydrogenase; HSPD1, heat shock $60 \mathrm{kDa}$ protein 1 (chaperonin). 
or death by any cause or to the end of follow-up (date of last contact with the treating physician). Taking into account the possible biological effects of the selected genes/ variants on cancer progression and a possible correlation between the variants, correction for multiple comparisons was excluded.

\section{Results}

SNP selection

A total of 29 SNPs were identified in the regions of interest (5' - and 3'-UTR, promoter and non-synonymous coding SNPs) of the seven genes NDUFS2, UQCRB, COX5B, COX6B1, ATP5C1, GAPDH and HSPD1 (Additional file 1: Table S1). Out of them, 18 SNPs were selected for the association studies, covering all gene regions with a putative regulatory function.

\section{Association with survival}

The two polymorphisms in the 3'-UTR region of UQCRB (complex III) showed association with CRC survival (Table 3 and Additional file 1: Table S2). The marker rs7836698 was statistically significantly associated with a decreased risk of death due to CRC $(\mathrm{HR}=0.53,95 \% \mathrm{CI}$ $0.31-0.91$ for TT), or due to any cause ( $\mathrm{HR}=0.52,95 \% \mathrm{CI}$ 0.32-0.85). For rs10504961, only the association with overall survival was statistically significant $(\mathrm{HR}=0.64,95 \%$ CI $0.42-0.99$ for TT). Interestingly, for both UQCRB polymorphisms, the TT carriers were more likely to develop tumours in the colon than in the rectum, with ORs of 2.02 (95\% CI 1.24-3.28) for rs7836698 (TT vs. CC) and 1.74 (95\% CI 1.08-2.78) for rs10504961 (TT vs. CC). When survival was analysed according to the cancer site, both SNPs showed association exclusively in the group of patients with cancer located in the colon (HR for overall survival $0.42,95 \%$ CI $0.22-0.82$ for rs7836698 TT carriers and 0.46 , 95\% CI $0.25-0.83$ for rs10504961 TT carriers), but not in the rectum (Table 3). The Kaplan-Meier survival curves representing the survival rates of the patients according to their genotypes, as well as the $\mathrm{P}$ values for the log-rank test are presented in Figure 1.

We examined also whether inclusion of other variables associated with CRC survival affected the parameter estimates for the UQCRB polymorphisms. The risk of death for carriers of the TT genotype at rs7836698 remained statistically significantly decreased after adjustment for age at diagnosis and TNM stage. Among patients with tumour located in the colon, the hazard ratios were similar both in the uni- and multivariate analyses for rs7836698 and rs10504961, although the statistical significance was sometimes lost.

\section{Association with CRC progression}

Three of the 16 SNPs investigated showed an association with one of the different components that form the UICC

Table 3 Association of two polymorphisms in UQCRB (complex III) with survival

\begin{tabular}{|c|c|c|c|c|c|c|c|c|}
\hline \multirow[t]{2}{*}{ SNP } & \multirow[t]{2}{*}{ Genotype } & \multicolumn{4}{|c|}{ Cause of death: CRC } & \multicolumn{3}{|c|}{ Cause of death: any } \\
\hline & & No. & Deaths (\%) & $\mathrm{HR}(95 \% \mathrm{Cl})$ & $P$ value & Deaths (\%) & $\mathrm{HR}(95 \% \mathrm{Cl})$ & $P$ value \\
\hline \multirow[t]{3}{*}{ rs7836698All patients } & CC & 203 & $54(26.6)$ & 1.00 & & $69(34.0)$ & 1.00 & \\
\hline & CT & 297 & $77(25.9)$ & $0.90(0.63-1.28)$ & 0.55 & $95(32.0)$ & $0.87(0.64-1.20)$ & 0.41 \\
\hline & $\Pi$ & 106 & $18(17.0)$ & $0.53(0.31-0.91)$ & 0.02 & $23(21.7)$ & $0.52(0.32-0.85)$ & 0.01 \\
\hline \multirow[t]{3}{*}{ rs7836698Colon } & CC & 97 & $30(30.9)$ & 1.00 & & $37(38.1)$ & 1.00 & \\
\hline & CT & 159 & $38(23.9)$ & $0.74(0.45-1.21)$ & 0.23 & 47 (29.6) & $0.75(0.48-1.18)$ & 0.21 \\
\hline & $\pi$ & 69 & $10(14.5)$ & $0.42(0.21-0.87)$ & 0.02 & $13(18.8)$ & $0.42(0.22-0.82)$ & 0.01 \\
\hline \multirow[t]{3}{*}{ rs7836698Rectum } & CC & 105 & $23(21.9)$ & 1.00 & & $31(29.5)$ & 1.00 & \\
\hline & $\mathrm{CT}$ & 137 & $39(28.5)$ & $1.13(0.67-1.90)$ & 0.65 & $48(35.0)$ & $1.03(0.65-1.63)$ & 0.89 \\
\hline & $\pi$ & 37 & $8(21.6)$ & $0.73(0.32-1.71)$ & 0.47 & $10(27.0)$ & $0.70(0.33-1.47)$ & 0.34 \\
\hline \multirow[t]{3}{*}{ rs10504961All patients } & CC & 134 & $34(25.4)$ & 1.00 & & $47(35.1)$ & 1.00 & \\
\hline & CT & 319 & $78(24.4)$ & $0.88(0.58-1.33)$ & 0.54 & $97(30.4)$ & $0.79(0.55-1.13)$ & 0.19 \\
\hline & $\pi$ & 150 & $36(24.0)$ & $0.76(0.47-1.24)$ & 0.28 & $42(28.0)$ & $0.64(0.42-0.99)$ & 0.05 \\
\hline \multirow[t]{3}{*}{ rs10504961Colon } & CC & 64 & $22(34.4)$ & 1.00 & & $27(42.2)$ & 1.00 & \\
\hline & $\mathrm{CT}$ & 168 & $38(22.6)$ & $0.61(0.35-1.05)$ & 0.07 & $48(28.6)$ & $0.62(0.38-1.01)$ & 0.06 \\
\hline & $\pi$ & 92 & 18 (19.6) & $0.47(0.25-0.90)$ & 0.02 & $22(23.9)$ & $0.46(0.25-0.83)$ & 0.01 \\
\hline \multirow[t]{3}{*}{ rs10504961Rectum } & $\mathrm{CC}$ & 70 & $12(17.1)$ & 1.00 & & $20(28.6)$ & 1.00 & \\
\hline & CT & 149 & $39(26.2)$ & $1.33(0.69-2.54)$ & 0.39 & $48(32.2)$ & $0.98(0.58-1.66)$ & 0.95 \\
\hline & $\pi$ & 58 & $18(31.0)$ & $1.41(0.66-2.97)$ & 0.37 & $20(34.5)$ & $0.96(0.51-1.81)$ & 0.89 \\
\hline
\end{tabular}

$\mathrm{HR}$, hazard ratio; $\mathrm{Cl}$, confidence interval. 


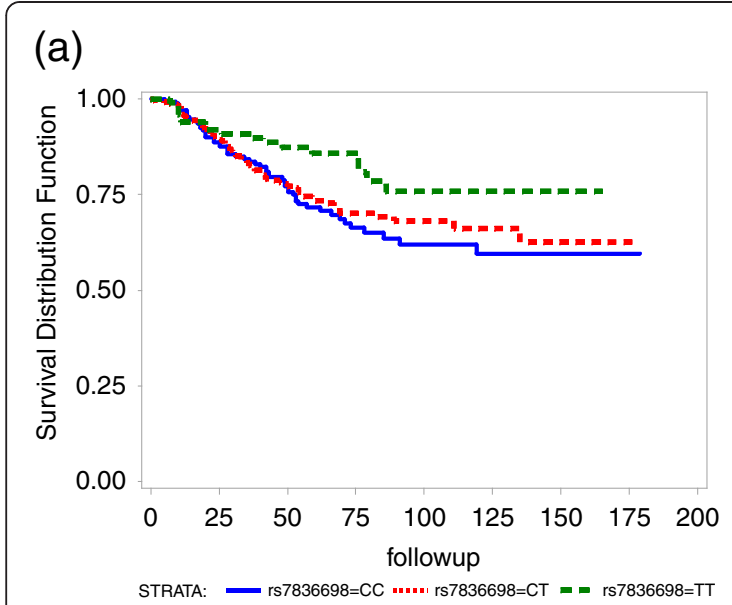

(c)

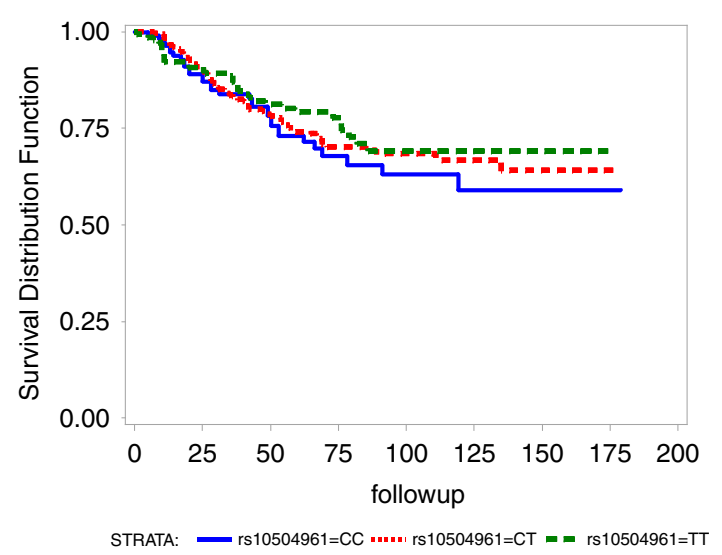

(b)

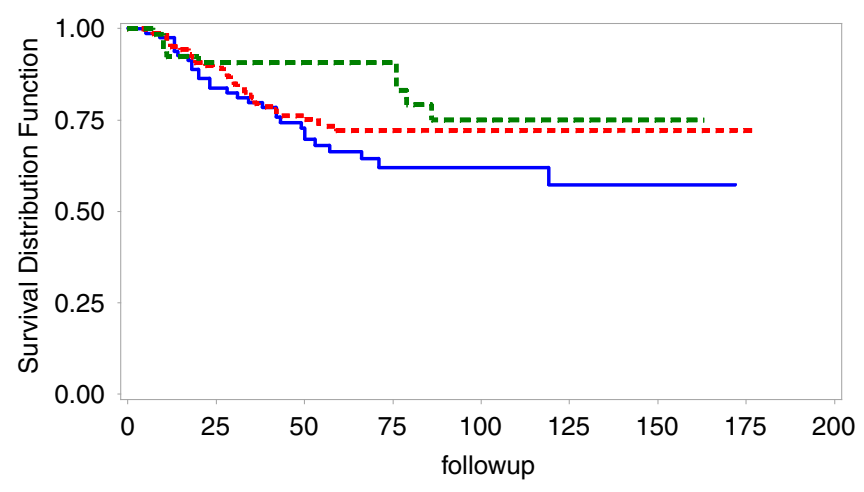

(d)

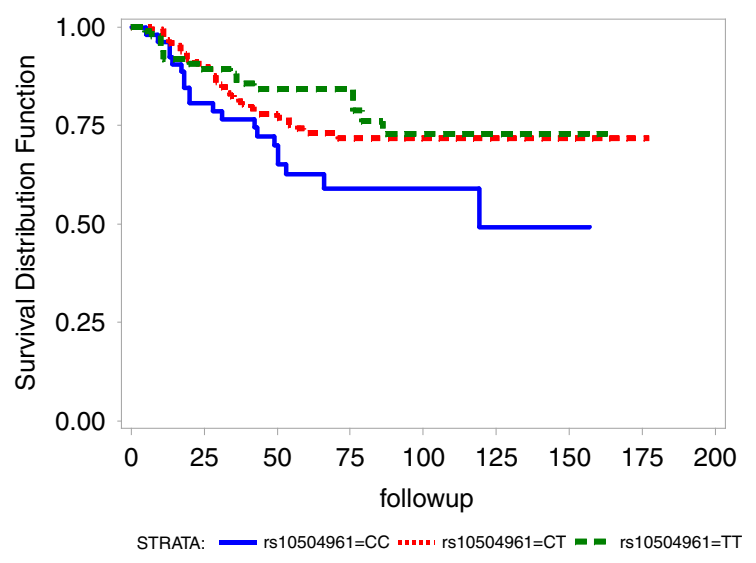

Figure 1 Kaplan-Meier estimates of CRC specific survival (months) according to UQCRB genotypes, (a) rs7836698, all CRC patients $(n=606)$, log rank $p$ value $=0.07$, (b) rs7836698, all patients diagnosed with colon cancer $(n=324)$, $\log$ rank $p$ value $=0.05$, (c) rs10504961, all CRC patients $(n=606)$, log rank $p$ value $=0.55$, and $(d)$ rs 10504961 , all patients diagnosed with colon cancer $(n=324)$, log rank $p$ value $=0.06$.

stage (T, N and $M$ ) in a dominant model. Two SNPs in COX6B1 (complex IV) showed an association with the presence of affected lymph nodes (rs6510502, OR $=1.75$, 95\% CI 1.20-2.57; rs10420252, OR=1.68, 95\% CI 1.112.53). The polymorphism rs6510502 was also associated with distant metastasis (OR $=1.67,95 \%$ CI 1.09-2.56). The marker rs7971637 in GAPDH was associated with tumour stage T3/T4 $(\mathrm{OR}=1.60,95 \%$ CI 1.11-2.30).

\section{Discussion}

In a homogeneous Northern German population of 613 CRC patients, we observed several associations of polymorphisms in the OXPHOS chain with survival and progression of the disease. Mainly, two SNPs in the 3'-UTR region of UQCRB (complex III), rs7836698 and rs10504961, were associated with survival. The two polymorphisms were in relatively strong linkage disequilibrium
$\left(D^{\prime}=0.98, r^{2}=0.65\right.$ in the 613 samples), explaining the association observed with the two SNPs. Added to that, the two studied polymorphisms in COX6B1 (complex IV), rs6510502 in the promoter and rs10420252 in the $5^{\prime}$ UTR, were associated with lymph node metastasis, and rs6510502 also with distant metastasis. The two markers were in high $\operatorname{LD}\left(\mathrm{D}^{\prime}=0.97, \mathrm{r}^{2}=0.71\right.$ in the 613 samples).

Patients carrying the TT genotype for any of the two SNPs in the UQCRB gene had an increased CRC survival. Interestingly, the association appeared to be restricted to the group of patients with tumour located in the colon. Multivariate analyses confirmed that the association was independent of other variables associated with CRC survival, indicating that both markers might act as independent prognostic markers. In the group of patients with tumour located in the colon the hazard ratios remained low, even though the significance 
sometimes disappeared when other variables were included. Our results may partially explain the previously reported higher familial risk for colon than for rectal cancer, which was shown to decrease gradually from proximal colon to distal colon and to rectum [17]. Also for survival, differences have been observed between colon and rectal cancer, with rectal cancer having somewhat better survival compared to colon cancer [18].

UQCRB is one of the ten nucleus-encoded subunits of the complex III in the mitochondrial respiratory chain, the ubiquinol-cytochrome $c$ oxidoreductase. In a genome-wide analysis of pancreatic cancer with CGH (comparative genomic hybridization) arrays, a DNA copy number variation was identified at the UQCRB locus, and this gene was suggested as a potential diagnostic marker or a therapeutic target [19]. Recently, it has been demonstrated that UQCRB plays a crucial role in the oxygen sensing mechanism that regulates responses to hypoxia. Using the small molecule terpestacin to inhibit UQCRB, tumour angiogenesis was blocked in vivo [20]. COX6B1 is one of the ten nucleus-encoded subunits of the complex IV in the mitochondrial respiratory chain, the cytochrome c oxidase. Mutations in this gene have been linked to severe infantile encephalomyopathy, a phenotype associated with cytochrome c oxidase deficiency [21]. Our results suggest that genetic variation in the regulatory regions of genes in the OXPHOS chain may associate with CRC progression. Whether such variation affects the gene expression pattern, impairs the function of mitochondria and leads to cancer development and progression remains to be elucidated.

One of the polymorphisms we investigated, rs2802460, located on the promoter of ATP5C1 (complex V), was reported to regulate expression of the gene in lymphoblastoid cell lines in a genome-wide association study of global gene expression [15]. However, this SNP did not show any association with CRC survival or disease progression. A recent report has evaluated the role of 376 tagSNPs (one included in our study, rs11576415 in NDUFS2) in 78 nuclear-encoded mitochondrial genes (including ATP5C1, NDUFS2, and UQCR) in prostate cancer risk, without clear evidence of association [22]. Another recent large association study included variants from 90 genes involved in oxidative phosphorylation (including ATP5C1, COX5B, COX6B1, GAPDH, and NDUFS2) and tested their association with epithelial ovarian cancer risk; no indication of association was found [23]. To our knowledge, this is the first association study of variants in the respiratory chain with CRC survival and progression.

\section{Conclusions}

Our findings suggest a possible influence of genes in the OXPHOS chain in CRC survival and progression, and support the newly discovered Warburg's hypothesis. This is the first report of a possible role of genes in the respiratory chain in the prognosis of CRC patients, but further independent studies are clearly needed to elucidate the validity of the results.

\section{Additional file}

Additional file 1: Table S1. SNPs in the regions of interest (5'- and 3'-UTR, promoter region and non-synonymous coding SNPS) with MAF $>0$ in European population. Table S2. Association of the 16 investigated polymorphisms with survival.

Competing interests

The authors declare that they have no competing interests.

\section{Author's contributions}

$J, K H$, and AF conceived and designed the study. $J$ carried out the molecular genetic studies. JL wrote the initial manuscript. MB performed the statistical analyses. $\mathrm{KH}$ and $\mathrm{AF}$ provided oversight and conceptual guidance to the project. MB, WS, HK, SB, JH, KH, AF and CS contributed to the final manuscript. WS, HK, HA, JB, JE, SB, TB, SS, JH, and CS participated in the clinical survey and sample collection. All authors read and approved the final manuscript.

\section{Acknowledgement}

This work was supported by the German National Genome Research Network (NGFN-Plus) [01GS08181]; the Deutsche Krebshilfe (German Cancer AID) [107318]; and European Union (EU) [HEALTH-F4-2007-200767].

\section{Author details}

'Division of Molecular Genetic Epidemiology, German Cancer Research Center (DKFZ), Im Neuenheimer Feld 580, Heidelberg, 69120, Germany. ${ }^{2}$ Department of General and Thoracic Surgery, Christian-Albrechts-University, Kiel, Germany. ${ }^{3}$ Division of Molecular Oncology, Institute for Experimental Cancer Research, Christian-Albrechts-University, Kiel, Germany. ${ }^{4}$ POPGEN Biobank Project, Christian-Albrechts-University, Kiel, Germany. ${ }^{5}$ Department of General Internal Medicine, Christian-Albrechts-University, Kiel, Germany. ${ }^{6}$ Center for Primary Health Care Research, Clinical Research Center, Lund University, Malmö, Sweden.

Received: 20 October 2011 Accepted: 30 April 2012

Published: 30 April 2012

\section{References}

1. Ferlay J, Shin HR, Bray F, Forman D, Mathers C, Parkin DM: Estimates of worldwide burden of cancer in 2008: GLOBOCAN 2008. Int J Cancer 2010, 127(12):2893-2917.

2. Walther A, Johnstone E, Swanton C, Midgley R, Tomlinson I, Kerr D: Genetic prognostic and predictive markers in colorectal cancer. Nat Rev Cancer 2009, 9(7):489-499.

3. Lascorz J, Chen B, Hemminki K, Forsti A: Consensus pathways implicated in prognosis of colorectal cancer identified through systematic enrichment analysis of gene expression profiling studies. PLoS One 2011, 6(4):e18867.

4. Warburg O: On respiratory impairment in cancer cells. Science 1956, 124 (3215):269-270.

5. Levine AJ, Puzio-Kuter AM: The control of the metabolic switch in cancers by oncogenes and tumor suppressor genes. Science 2010, 330 (6009):1340-1344.

6. Cuezva JM, Sanchez-Arago M, Sala S, Blanco-Rivero A, Ortega AD: A message emerging from development: the repression of mitochondrial beta-F1-ATPase expression in cancer. J Bioenerg Biomembr 2007, 39 (3):259-265.

7. Ristow M: Oxidative metabolism in cancer growth. Curr Opin Clin Nutr Metab Care 2006, 9(4):339-345.

8. Koppenol WH, Bounds PL, Dang CV: Otto Warburg's contributions to current concepts of cancer metabolism. Nat Rev Cancer 2011, 11(5):325-337. 
9. Eng C, Kiuru M, Fernandez MJ, Aaltonen LA: A role for mitochondrial enzymes in inherited neoplasia and beyond. Nat Rev Cancer 2003, 3 (3):193-202.

10. Chandra D, Singh KK: Genetic insights into OXPHOS defect and its role in cancer. Biochim Biophys Acta 2010, 1807(6):620-625.

11. Krawczak M, Nikolaus S, von Eberstein $\mathrm{H}$, Croucher PJ, El Mokhtari NE, Schreiber S: PopGen: population-based recruitment of patients and controls for the analysis of complex genotype-phenotype relationships. Community Genet 2006, 9(1):55-61.

12. Castro FA, Forsti A, Buch S, Kalthoff H, Krauss C, Bauer M, Egberts J, Schniewind B, Broering DC, Schreiber S, et al: TLR-3 polymorphism is an independent prognostic marker for stage II colorectal cancer. Eur J Cancer 2011, 47(8):1203-1210.

13. Kanehisa M, Goto S, Kawashima S, Okuno Y, Hattori M: The KEGG resource for deciphering the genome. Nucleic Acids Res 2004, 32:D277-D280 (Database issue).

14. Barrett JC, Fry B, Maller J, Daly MJ: Haploview: analysis and visualization of LD and haplotype maps. Bioinformatics 2005, 21(2):263-265.

15. Dixon AL, Liang L, Moffatt MF, Chen W, Heath S, Wong KC, Taylor J, Burnett E, Gut I, Farrall M, et al: A genome-wide association study of global gene expression. Nat Genet 2007, 39(10):1202-1207.

16. Adzhubei IA, Schmidt S, Peshkin L, Ramensky VE, Gerasimova A, Bork P, Kondrashov AS, Sunyaev SR: A method and server for predicting damaging missense mutations. Nat Methods 2010, 7(4):248-249.

17. Hemminki $K$, Chen B: Familial risks for colorectal cancer show evidence on recessive inheritance. Int J Cancer 2005, 115(5):835-838

18. Storm HH, Engholm G, Hakulinen T, Tryggvadottir L, Klint A, Gislum M, Kejs AM, Bray F: Survival of patients diagnosed with cancer in the Nordic countries up to 1999-2003 followed to the end of 2006. A critical overview of the results. Acta Oncol 2010, 49(5):532-544.

19. Harada T, Chelala C, Crnogorac-Jurcevic T, Lemoine NR: Genome-wide analysis of pancreatic cancer using microarray-based techniques. Pancreatology 2009, 9(1-2):13-24.

20. Jung HJ, Shim JS, Lee J, Song YM, Park KC, Choi SH, Kim ND, Yoon JH, Mungai PT, Schumacker PT, et al: Terpestacin inhibits tumor angiogenesis by targeting UQCRB of mitochondrial complex III and suppressing hypoxia-induced reactive oxygen species production and cellular oxygen sensing. J Biol Chem 2010, 285(15):11584-11595.

21. Massa V, Fernandez-Vizarra E, Alshahwan S, Bakhsh E, Goffrini P, Ferrero I, Mereghetti P, D'Adamo P, Gasparini P, Zeviani M: Severe infantile encephalomyopathy caused by a mutation in COX6B1, a nucleusencoded subunit of cytochrome c oxidase. Am J Hum Genet 2008, 82 (6):1281-1289.

22. Wang L, McDonnell SK, Hebbring SJ, Cunningham JM, St Sauver J, Cerhan JR, Isaya G, Schaid DJ, Thibodeau SN: Polymorphisms in mitochondrial genes and prostate cancer risk. Cancer Epidemiol Biomarkers Prev 2008, 17 (12):3558-3566.

23. Permuth-Wey J, Chen YA, Tsai YY, Chen Z, Qu X, Lancaster JM, Stockwell H, Dagne $G$, Iversen E, Risch $H$, et al: Inherited variants in mitochondrial biogenesis genes may influence epithelial ovarian cancer risk. Cancer Epidemiol Biomarkers Prev 2011, 20(6):1131-1145.

doi:10.1186/1471-2350-13-31

Cite this article as: Lascorz et al:: Polymorphisms in the mitochondrial oxidative phosphorylation chain genes as prognostic markers for colorectal cancer. BMC Medical Genetics 2012 13:31.

\section{Submit your next manuscript to BioMed Central and take full advantage of:}

- Convenient online submission

- Thorough peer review

- No space constraints or color figure charges

- Immediate publication on acceptance

- Inclusion in PubMed, CAS, Scopus and Google Scholar

- Research which is freely available for redistribution 